\title{
Association between Phase Coupling of Respiratory Sinus Arrhythmia and Brain Activity during Sleep
}

\author{
Kyuichi Niizeki, Narumi Ukawa, Tadashi Saitoh \\ Biosystems Engineering, Graduate School of Science and Engineering, Yamagata University \\ Johnan 4-3-16, Yonezawa, Japan \\ nzq@yz.yamagata-u.ac.jp; uka@yz.yamagata-u.ac.jp; saitoh-t@yz.yamagata-u.ac.jp
}

\section{Extended Abstract}

Our previous study [1] demonstrated that mental stress in humans exerts an influence on the oscillations of respiratory sinus arrhythmia (RSA), inducing an incoherent phase lag with respect to breathing, i.e., decrease in the phase coherence ( $\square$ ) between RSA and respiration. The $\square$ showed a positive correlation with high-frequency component (HF) of the frequency domain measures of heart rate variability (HRV) and a negative correlation with sympathovagal balance (lowfrequency to HF; LF/HF), suggesting that the phase coupling of RSA could be used as a noninvasive measure for evaluating the activity of the autonomic nervous system (ANS). We hypothesised that similar to the mental stress condition, would be increased during non-REM sleep as reflecting cardiac parasympathetic activation, since it has been demonstrated a strong interaction between ANS activity and delta wave activity in the EEG during sleep [2][3]. The aim of the present study was to examine how $\square$ is altered depending on the sleep status and to evaluate the temporal relationship between brain activity and $\square$ during sleep. Overnight EEG and ECG of seventeen healthy volunteers were recorded. The EEG was band-pass filtered to extract the activity in the delta, theta, alpha and beta ranges, and then Hilbert transform was applied to each extracted signal to calculate the analytic amplitude. Respiratory signal was derived from the amplitude modulation of R-waves caused by breathing. Beat-to-beat R-R intervals (RRI) were determined and the time series of the RRI was further band-pass filtered with a frequency range of $0.15-0.4 \mathrm{~Hz}$ to determine RSA. From the oscillatory signals of RSA and respiration, each analytic signal was constructed from a real signal and its Hilbert transform, and then the timedependent $\square$ between RSA and respiration was obtained with 10-s window.

Using auto- and cross-correlation analyses, we found that periodicity of $\square$ was quite similar to that of delta wave (93.3 min vs. $90.8 \mathrm{~min}, \mathrm{r}=0.900$ ) and that overnight profiles of $\square$ and delta wave were related with highly significant crosscorrelation coefficients $(0.46 \pm 0.11)$. The variation of $\square$ preceded changes in delta wave by 7.6 min on average. These results demonstrate that $\square$ and EEG delta activity are closely linked during sleep in healthy humans and suggest that the phase coupling analysis of RSA could provide important information on sleep status and cardiac autonomic modulation during sleep.

\section{References}

[1] K. Niizeki and T. Saitoh, "Incoherent oscillations of respiratory sinus arrhythmia during acute mental stress in humans," Am J Physiol Heart Circ Physiol. vol. 302, pp. H359-H367, 2012.

[2] G. Brandenberger, J. Ehrhart, F. Piquard, C. Simon, "Inverse coupling between ultradian oscillations in delta wave activity and heart rate variability during sleep," Clin Neurophysiol., vol. 112, pp. 992-996, 2001.

[3] F. Jurysta, P. van de Borneb, P. F. Migeottec, M. Dumontd, J. P. Lanquarta, J. P. Degauteb, P. Linkowskia, “A study of the dynamic interactions between sleep EEG and heart rate variability in healthy young men," Clin Neurophysiol., vol. 114, pp. 2146-2155, 2003. 